\title{
La Incidencia de Metales Pesados en Compost de Residuos Sólidos Urbanos y en su uso Agronómico en España
}

Antonio Rosal, Jose P. Pérez, María A. Arcos y Manuel Dios

Universidad de Córdoba, Departamento de Ingeniería Química,Campus Universitario de Rabanales, Carretera N-IV, Km. 396, Edif. Marie Curie, 14071 Córdoba - España (e-mail: manolodios@uco.es)

\begin{abstract}
Resumen
En este trabajo, se describe el proceso de compostaje mediante pilas volteadas de la fracción orgánica, recogida selectivamente, de residuos sólidos urbanos (RSU), analizado durante tres años. El compost, aceptable para su uso agrícola, fue aplicado en cultivos de trigo y ajo, combinando tratamientos mixtos con el objetivo de conocer que tipo de fertilización era la que generaba los mejores resultados, y demostrar como la fertilización orgánica puede llegar a ser una alternativa real en nuestros suelos con los consiguientes beneficios ambientales. No se encontraron incidencias de metales sobre los frutos ni los suelos. La producción fue también semejante al control. A pesar de todo esto, en caso de aprobación de la nueva Normativa Europea que se encuentra en fase de discusión, y con las restricciones que se pretenden imponer, el compost de RSU fabricado en la ciudad de Córdoba (España) tendrá grandes dificultades para su uso agrícola si se continúan con las mismas prácticas de gestión debido a su alto contenido en metales.
\end{abstract}

Palabras clave: compost de RSU, metales pesados, uso agrícola, fertilización agromineral

\section{Impact of Heavy Metals in Composting of Municipal Solid Wastes and its use in Agriculture in Spain}

\begin{abstract}
In this work, our group do a research about the composting process, in turned windrow, of the organic fraction of the municipal solid waste (MSW), selectively collected, that was analysed for three years. Suitable compost for agricultural use was obtanied. This compost was used in wheat and garlic farming. No incidence of metals on fruit and soil was detected. Crops were found to bew similar to thoses observed for a control test. However, requirements in metal contents are increasing in European Union rules. Therefore, this Compost of Municipal Solid Waste made in Cordoba (Spain) will not be accepted for an agricultural use with the actual management.
\end{abstract}

Keywords: MSW compost, heavy metals, agricultural use, organomineral fertilization 


\section{INTRODUCCIÓN}

Si bien son escasas aún las regulaciones especificas sobre la gestión de residuos biodegradables en Europa, una Directiva del año 1999 sobre depósito de residuos en vertederos estipula una progresiva reducción en el vertido de materia orgánica en éstos. Frente a este problema, una de las posibles opciones, probablemente la más recomendada en países con una necesidad elevada de materia orgánica en sus suelos, es el compostaje. La producción de compost debe ser considerada como una parte integrada en todo el proceso de tratamiento de los residuos y como una opción dentro de las tecnologías disponibles, tales como digestión anaerobia, el reciclado de lodos, la incineración o el depósito final en vertederos de seguridad. La directiva europea 1999/31/CE estipula importantes objetivos para la reducción de residuos biodegradables que finalmente acabaran en vertederos. Los objetivos son:

- La reducción a un $75 \%$ en peso de total de residuos biodegradables municipales de los producidos en 1995 para el año 2006.

- La reducción de estos residuos al 50\% para el 2009.

- La reducción al 35\% para el 2016.

El compost es ampliamente utilizado para mejorar la estructura del suelo, estimular su actividad micróbica, incrementar su contenido en carbono y añadir nitrógeno y otros nutrientes al mismo (Giusquiani et al., 1995, Parkinson et al., 1999, Karaca et al., 2006). Por otra parte, el parcial reemplazo de la turba utilizada en semilleros por compost se ha incrementado de manera importante en pocos años, lo que requiere un buen conocimiento de la estabilidad de la materia orgánica y la dinámica de sus nutrientes (Abad et al., 2001, Papafotiou et al., 2004, Agehara y Warnke, 2005). Tan solo el alto contenido en sales de algunos compost desaconseja su uso para este uso tan específico (Moldes et al., 2007). A pesar de sus ventajas, su utilización no está exenta de riesgos, sobre todo los derivados del uso de materia orgánica no estabilizada. Los principales son; la reducción de oxígeno radicular, la producción de compuestos fitotóxicos en el suelo o la inmovilización del nitrógeno por los microorganismos en enmiendas con alta relación C/N (Bengtsson et al., 2003). Esto es especialmente importante cuando son usados para el reemplazo de turbas en medios de crecimiento (lannotti et al., 1994).

Junto a sus propiedades agronómicas, el compost ayuda a mitigar grandes impactos ambientales de repercusión global, como el calentamiento global (Power y Murphy, 2006) y es la opción mas respetuosa con el medio si se compara con el vertido directo, la estabilización para vertido por tratamientos mecánicos-biológicos o la incineración (Hong et al., 2006). Soluciona parcialmente el problema de vertido de residuos urbanos y además, el producto generado actúa como un sumidero de carbono, y por tanto contribuye a la reducción del efecto invernadero. Por último, actúa como una enmienda orgánica en la restauración de suelos amenazados por la erosión y afectados en mayor o menor medida por algún tipo de degradación. No obstante, ciertas medidas de seguridad o valores límites recomendados deben tenerse en cuenta en la aplicación de compost para un correcto equilibrio suelo-planta.

El segundo documento de trabajo que ejerce de borrador de directiva sobre el tratamiento de residuos biodegradables ( $2^{\text {nd }}$ Draft of Biological Treatment of Biowaste) del año 2001 intenta hacer una clara distinción entre "Compost", derivado de Residuos orgánicos y "Bio-residuo estabilizado". En esta línea, el compost sería usado y comercializado como producto de calidad, apto para todas las áreas de producción de alimentos, semilleros y jardinería, mientras que el bio-residuo estabilizado, concebido como un pretratamiento de los residuos con objeto de reducir su biodegradabilidad y en consecuencia su peligrosidad, solo se recomendaría para usos marginales o la restauración paisajística. La distinción entre ambas categorías se hace en base al contenido en metales, que centra el estudio que aquí se presenta.

En este trabajo, se describe el proceso de compostaje mediante pilas volteadas de la fracción orgánica, recogida selectivamente, de residuos sólidos municipales, el cual fue analizado durante tres años. El proceso de compostaje y el compost producido ha sido caracterizado. Asimismo, se ha estudiado la incidencia de los metales pesados en la capa arable de suelo tras la aplicación de compost y se han comparado las cosechas obtenidas con fertilizantes minerales. 


\section{MATERIALES Y MÉTODOS}

El proceso de compostaje mediante pilas volteadas de la fracción orgánica se realizó a escala industrial y se repitió durante tres años.

Una pila de compost, con 20 toneladas de peso, fue periódicamente volteada y humedecida en caso necesario durante 140 días hasta garantizar su estabilidad biológica (Chica et al., 2003, Grigatti et al., 2007). Finalmente, el compost refinado, con un tromel de $15 \mathrm{~mm}$ de luz y mesa densimétrica, fue obtenido cada año y dispuesto para su uso agrícola en experimentos anuales.

En cada experimento, se recogió una muestra de $500 \mathrm{~g}$ de peso de diferentes partes de la pila que se utilizó para las caracterizaciones químicas. A cada extracto acuoso, se le determinó el pH, usando un pH-metro CRISON Micro pH 2002, así como la Conductividad Eléctrica (CE), que fue determinada usando un conductimetro CRISON Micro CM 2200. A la materia seca, secada a $60^{\circ} \mathrm{C}$ durante 72 horas, se le determinó el contenido en nitrógeno, que fue medido usando el Método del Nitrógeno Total Kjeldahl (APHA, 1998); la materia orgánica fue determinada usando el método del dicromato (APHA, 1989); el contenido en fósforo fue medido mediante espectrofotometría usando un espectrofotómetro BECKMAN DU 640. Finalmente, la cantidad de metales fue determinada mediante un espectrofotómetro de absorción atómica con llama, PERKIN ELMER AA-300, tras una digestión ácida en $\mathrm{HCl} 2 \mathrm{~N}$. Las características químicas del material fresco (RSU) y del compost refinado se muestran en la Tabla 1.

El experimento se desarrolló en una finca agrícola experimental en la vega del Guadalquivir en el municipio de Córdoba. Esta región tiene un clima mediterráneo con influencia continental y del atlántico en sus precipitaciones. Los inviernos son tranquilos con pocas heladas y los veranos son calurosos con temperaturas que sobrepasan los $40^{\circ}$ con facilidad. Las precipitaciones tienen lugar en primavera y otoño presentando una fuerte sequía estival. Durante tres años se repitieron los experimentos entre los meses de Noviembre y Junio, alternando el cultivo de trigo con el cultivo de ajo durante estos tres años. El experimento fue una combinación de tres tipos de aplicaciones de fertilizantes (añadiendo $180 \mathrm{Kg} / \mathrm{ha}$ de nitrógeno) y un tratamiento de referencia (Control, sin Nitrógeno). Se hicieron 4 repeticiones de cada uno de los tratamientos en las parcelas con la rotación trigo-ajo-trigo y 4 repeticiones de cada una en parcelas con rotación ajo-trigo-ajo cada año. Por tanto, se estudiaron 32 parcelas de $4 \times 10$ metros anualmente.

Conociendo los beneficios de la fertilización organomineral sobre las cosechas (Tejada et al., 1996) y sobre la dinámica del nitrógeno (Tejada et al., 2002), se diseñaron tres estrategias de fertilización: $100 \%$ de Nitrógeno Mineral (N-MINERAL), 100\% de compost de RSU (COMPOST), y una fertilización mixta al 50\%, entre Nitrógeno mineral y Nitrógeno de Compost de RSU (50C-50M).

Tabla 1: Características Químicas del material fresco (RSU) y Compost refinado en cada año

\begin{tabular}{ccccccc}
\hline & $\mathrm{pH}$ & $\mathrm{CE}\left(\mathrm{dS} \mathrm{m} \mathrm{m}^{-1}\right)$ & $\mathrm{M} . \mathrm{O} .(\%)$ & $\mathrm{N}_{\text {kjeldahl }}(\%)$ & $\mathrm{C} / \mathrm{N}$ & $\mathrm{P}_{\left(\% \mathrm{P}_{2} \mathrm{O}_{5}\right)}$ \\
\hline RSU & 6.1 & 8.0 & 58 & 2.4 & 14.1 & 0.3 \\
Compost 1 $^{\text {er }}$ año & 8.3 & 19.7 & 39 & 2.9 & 7.7 & 0.2 \\
Compost 2 $^{\circ}$ año & 8.4 & 13.2 & 36 & 2.6 & 8.1 & 0.3 \\
Compost 3 $^{\text {er }}$ año & 8.1 & 11.4 & 33 & 2.7 & 7.1 & 0.2
\end{tabular}

\section{RESULTADOS Y DISCUSIÓN}

La Tabla 2 muestra el contenido en metales del Residuo fresco (RSU) y el compost refinado cada año, comparándolo con los límites propuestos en la normativa Española en vigor. Todo el Compost producido satisface los límites legales en cuanto al máximo contenido metálico, aunque este compost quedaría clasificado como de tercera categoría.

Para corregir la falta de calidad que pudo desprestigiar a esta enmienda en el pasado y a falta de una regulación Europea del Compost son los estados miembros los que están realizando una 
legislación cada vez más restrictiva. En España, con objeto de proteger el medio ambiente y la salud pública para productos en cuya composición intervengan materias primas de origen animal o vegetal, el Real Decreto Español 824/2005 fija unos estrictos límites en metales pesados estableciendo hasta tres clases de calidad según la cantidad de estos últimos en el producto, e impone restricciones al uso del Compost de peor calidad, la Clase C. Con respecto a la anterior orden (de 28 de Mayo de 1998 y 2 de noviembre de 1999) no existen grandes variaciones en los contenidos exigibles de nitrógeno, materia orgánica, humedad, granulometría, plásticos e inertes, así como el contenido en Salmonella y E.Colli.

Tabla 2: Contenido en Metales en RSU, Compost y límites propuestos en diferentes normativas

\begin{tabular}{|c|c|c|c|c|c|c|}
\hline & $\begin{array}{c}\mathrm{Cd} \\
(\mathrm{mg} / \mathrm{Kg})\end{array}$ & $\begin{array}{c}\mathrm{Cu} \\
(\mathrm{mg} / \mathrm{Kg})\end{array}$ & $\begin{array}{c}\mathrm{Cr} \\
(\mathrm{mg} / \mathrm{Kg})\end{array}$ & $\begin{array}{c}\mathrm{Ni} \\
(\mathrm{mg} / \mathrm{Kg})\end{array}$ & $\begin{array}{c}\mathrm{Pb} \\
(\mathrm{mg} / \mathrm{Kg})\end{array}$ & $\begin{array}{c}\mathrm{Zn} \\
(\mathrm{mg} / \mathrm{Kg})\end{array}$ \\
\hline RSU & 4 & 47 & 104 & 98 & 120 & 90 \\
\hline Compost $1{ }^{\mathrm{er}}$ año & 3 & 276 & 30 & 50 & 165 & 415 \\
\hline Compost $2^{\circ}$ año & 3 & 252 & 57 & 57 & 120 & 579 \\
\hline $\begin{array}{l}\text { Compost } 3^{\text {er }} \text { año } \\
\text { Ley Española }\end{array}$ & 2 & 373 & 84 & 64 & 144 & 603 \\
\hline $\mathrm{RD} \mathrm{824/2005-A}$ & 0.7 & 70 & 70 & 25 & 45 & 200 \\
\hline RD 824/2005 - B & 2 & 300 & 250 & 90 & 150 & 500 \\
\hline RD 824/2005 - C & 3 & 400 & 300 & 100 & 250 & 1000 \\
\hline$\frac{\text { Etiqueta ecológica }}{\text { Decisión 2001/688/CE }}$ & 1.5 & 75 & 140 & 50 & 140 & 300 \\
\hline 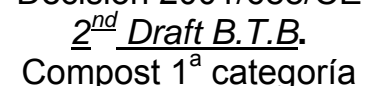 & 0.7 & 100 & 100 & 50 & 100 & 200 \\
\hline Compost $2^{\mathrm{a}}$ categoría & 1.5 & 150 & 150 & 75 & 150 & 400 \\
\hline Bio-residuo estabilizado & 5 & 600 & 600 & 150 & 500 & 1500 \\
\hline
\end{tabular}

Las concentraciones de Cadmio, Cobre y Zinc, fueron muy altas y hacen imposible considerar estos compost como productos ecológicos en el ámbito Europeo. De todas formas, si el segundo borrador sobre Tratamiento de Residuos biodegradables (B.T.B.) es aprobado, el producto resultante del compostaje de RSU será considerado como "Bio-residuo Estabilizado" no apto para su uso agrícola. La incorporación de metales pesados a la materia orgánica procede en primer lugar de una segregación muy pobre que la población hace de los residuos en sus hogares. La escasa industrialización de esta ciudad permite afirmar que poca incidencia tiene este sector en la contribución de metales a la materia orgánica, por deposición atmosférica, contaminación de las aguas o residuos que en otros lugares influyen claramente (Dheri et al., 2007). Sin embargo, los inputs procedentes de la abrasión de superficies pintura, plomo y zinc de los materiales de construcción $u$ otros metales que están en la composición de productos de higiene contribuyen a estas altas concentraciones de metales en productos cotidianos.

Como el contenido en materia orgánica total durante el proceso de compostaje decrece por la respiración de la biomasa, que genera $\mathrm{CO}_{2}$ y $\mathrm{H}_{2} \mathrm{O}$, un incremento general en el contenido de metales es de esperar. Sin embargo, solo los contenidos en zinc y cobre experimentaron este aumento. En cierta medida, la bioacumulación por parte de la biomasa está implicada en este fenómeno (Premuzic et al., 2002) y la movilidad que tiene el Zinc (Xiaoli et al., 2007). Probablemente, el resto de los metales fueron parcialmente rechazados en el proceso de refinado y eliminación de restos inertes.

El contenido en metales para los suelos antes del primer experimento y al final de los tres años fue analizado y se muestra en la Tabla 3. Como podemos observar, existe un ligero incremento en la mayoría de los metales al cabo de los tres años aplicando compost, pero no hay diferencias significativas entre los diferentes tratamientos estudiados. Se estima que gran parte de los metales queda retenida en los suelos y que debido al alto contenido en arcilla y pH alcalino de los suelos, la movilidad y biodisponibilidad ha estado limitada, razonamiento que puede ser extrapolable a gran parte de los suelos de la cuenca Mediterránea (Peris et al., 2007). 
Las cosechas de trigo y ajo (Tabla 4) fueron diferentes después de cada año debido a razones climatológicas. Para cada año, los tratamientos con N-MINERAL y 50C-50M generan similares resultados y dan las más altas producciones. De todas formas, el tratamiento con COMPOST $(100 \%)$ genera unas producciones tan solo ligeramente mejores que las obtenidas por el CONTROL, probablemente por su alta conductividad eléctrica (Castillo et. al., 2004). Tampoco se encontraron diferencias significativas en tratamientos combinados ( $\mathrm{N}$-orgánico y $\mathrm{N}$-mineral) en experiencias realizadas en Italia en suelos de similares características y dentro de el área Mediterránea (Montemurro y Maiorana, 2007).

Tabla 3: Contenido en metales para la capa arable en el suelo $(0-15 \mathrm{~cm})$

\begin{tabular}{ccccccc}
\hline & $\mathrm{Cd}(\mathrm{mg} / \mathrm{Kg})$ & $\mathrm{Cu}(\mathrm{mg} / \mathrm{Kg})$ & $\mathrm{Cr}(\mathrm{mg} / \mathrm{Kg})$ & $\mathrm{Ni}(\mathrm{mg} / \mathrm{Kg})$ & $\mathrm{Pb}(\mathrm{mg} / \mathrm{Kg})$ & $\mathrm{Zn}(\mathrm{mg} / \mathrm{Kg})$ \\
\hline Antes exp. & 0.8 & 14 & 31 & 54 & 34 & 26 \\
CONTROL & 1.0 & 22 & 25 & 19 & 39 & 32 \\
N-MINERAL & 1.3 & 20 & 29 & 20 & 39 & 28 \\
5OC-50M & 1.5 & 24 & 21 & 22 & 36 & 32 \\
COMPOST & 1.5 & 25 & 32 & 20 & 42 & 33 \\
\hline
\end{tabular}

Tabla 4: Cosechas obtenidas de trigo y ajo

\begin{tabular}{ccccccc}
\hline & \multicolumn{3}{c}{ TRIGO $(\mathrm{Kg} / \mathrm{ha})$} & \multicolumn{3}{c}{ AJO $(\mathrm{Kg} / \mathrm{ha})$} \\
\cline { 2 - 7 } & $1^{\text {er }}$ año & $2^{\circ}$ año & $3^{\text {er }}$ año & $1^{\text {er }}$ año & $2^{\circ}$ año & $3^{\text {er }}$ año \\
\hline Antes exp. & 4450 & 4860 & 1340 & 2290 & 8130 & 15240 \\
CONTROL & 3930 & 5080 & 1960 & 2130 & 7470 & 14010 \\
N-Mineral & 3040 & 4500 & 1980 & 1820 & 6590 & 12350 \\
50C-50M & 2710 & 4340 & 1150 & 1620 & 5600 & 10490 \\
\hline
\end{tabular}

Por otra parte, no existen diferencias significativas en las concentraciones de metales en el grano de trigo y los bulbos de ajo producidos. Solo los contenidos en Cobre, Cromo y Zinc en la cosecha mas pobre del trienio para el trigo (la del tercer año) fueron más altos de $10 \mathrm{mg} / \mathrm{Kg}$. También se encontraron $2.3 \mathrm{mg} / \mathrm{Kg}$ de Cadmio y más de $10 \mathrm{mg} / \mathrm{Kg}$ del resto de metales en la cosecha más pobre de ajo en el primer año del trienio.

\section{CONCLUSIONES}

El Compost de Residuos Sólidos Urbanos hecho en Córdoba, ciudad española poco industrializada, satisface los límites actuales de la legislación vigente para su aplicación agrícola, los cuales son bastante restrictivos en bien de la seguridad alimentaría, y del medio ambiente. No obstante, en caso de desarrollarse nuevas restricciones sobre contenido en xenobioticos o determinación de la estabilidad biológica, este Compost difícilmente sería aceptado para un uso agrícola.

Los contenidos en Zinc y Cobre se incrementaron significativamente durante el proceso de compostaje ya que no se rechazan con el material inerte en el proceso de refinado del producto acabado. No se detectaron incidencias de metales sobre el grano de trigo y los bulbos de ajo.

Las cosechas obtenidas con la fertilización combinada 50C-50M fueron similares, o incluso en algunos casos mejores que aquellas obtenidas con Nitrógeno mineral, en parte debido a la incorporación de microbiota con el compost que favorece los procesos de nitrificación del Nitrógeno mineral añadido al suelo. Debido a que los suelos en los que se desarrolló este experimento gozaban de buena estructura y porosidad la aerobiosis estaba garantizada y los procesos de desnitrificación impedidos. El compost de RSU usado sólo, como enmienda, genera un desarrollo menos favorable en los cultivos.

\section{AGRADECIMIENTOS}

Al Ministerio de Educación y Ciencia por la financiación recibida a través del proyecto CTM 200501293 para la realización de este trabajo. 


\section{REFERENCIAS}

Abad M., P. Noguera y S. Bure's; National inventory of organic wastes for use as growing media for ornamental potted plant production: case study in Spain. Bioresource Technology 77 (2), 197-200 (2001).

Agehara S. y D.D. Warncke; Soil Moisture and Temperature Effects on Nitrogen Release from Organic Nitrogen Sources. Soil Science. Society American Journal, 69, 1844-1855 (2005).

APHA; Standard methods for the examination of water and wastewater. 17th edition. APHA Washington DC. (1989).

APHA; Standard methods for the examination of water and wastewater. 20th Edition. APHA Washington DC. (1998).

Bengtsson G., P. Bengtsson y K.F. Mansson; Gross nitrogen mineralization and nitrification rates as a function of soil C/N ratio and microbial activity. Soil Biology and Biochemistry 35 (1), 143-154 (2003).

Castillo J.E. y otros cinco autores; Municipal Solid Waste (MSW) compost as a tomato transplant medium. Compost Science and Utilization 12 (11), 86-92 (2004).

Chica A., J.J. Mohedo, M.A. Martín y A. Martín; Determination of the Stability of MSW compost using a respirometric technique. Compost Science and Utilization 11 (2), 169-175. (2003).

Dheri, G.S., M.S. Brar y S.S. Malhi; Heavy-metal concentration of sewage-contaminated water and its impact on underground water, soil, and crop plants in alluvial soils of northwestern, India. Communications in Soil Science and Plant Analysis 38 (9-10), 1353-1370 (2007).

Giusquiani P. y otros cuatro autores; Urban waste compost: effects on physical, chemical and biochemical soil properties. Journal of Environmental Quality 24, 175-182 (1995).

Grigatti M., M. Dios W. Blok y A. Veeken; A standardized method for the determination of the intrinsic carbon and nitrogen mineralization capacity of natural organic matter sources. Soil Biology and Biochemistry, 39, 1493-1503. (2007).

Hong, R.J. y otros seis autores; Life cycle assessment of BMT-based integrated municipal solid waste management: Case study in Pudong, China. Resources, Conservation and Recycling 49 (2), 129-146 (2006).

lannotti, D.A. y otros cuatro autores; Oxygen respirometry to assess stability and maturity of composted municipal solid waste. Journal of Environmental Quality 23, 1177-1183 (1994).

Karaca, A., O.C.Turgay y N.Tamer; Effects of a humic deposit (gyttja) on soil chemical and microbiological properties and heavy metal availability. Biology and Fertility of Soils 42 (6), 585-592 (2006).

Moldes, A., Y. Cendón y M.T. Barral; Evaluation of municipal solid waste compost as a plant growing media component, by applying mixture design. Bioresource Technology 98 (16), 3069-3075 (2007).

Montemurro F. y M. Maiorana; Nitrogen utilization, yield, quality and soil properties in a sugarbeet crop amended with municipal solid waste compost. Compost Science and Utilization 15 (2), 84-92 (2007).

Papafotiou, M. y otros cuatro autores; Olive-mill wastes compost as growing medium component for the production of poinsettia. Scientia Horticulturae 102 (2), 167-175 (2004). 
Parkinson, R., M. Fuller y A. Groenhof; An evaluation of greenwaste compost for the production of forage maize (Zea mays L.). Compost Science and Utilization 7 (2), 72-80 (1999).

Peris, M. y otros cuatro autores; Heavy metal contents in horticultural crops of a representative area of the European Mediterranean region. Science of the Total Environment, 378 (1-2), $42-48$ (2007).

Power, N.M. y J.D. Murphy; Composting of biodegradable municipal waste in Ireland. WIT Transactions on Ecology and the Environment 92, 303-312 (2006).

Premuzic, Z., J.P. Brichta, A.E. Rendina y A.F. Iorio; Parámetros de fertilidad y toxicidad para la comercialización como enmienda usando lodos del río Matanza en Argentina. Información Tecnológica, 13(5), 25-29 (2002).

Tejada, M., R. Baron, C. Benitez y J.L. Gonzalez; Variation of field crop characteristics as a result of organomineral fertilization during two years. Información Tecnológica, 7(5), 17-24 (1996).

Tejada, M., C. Benitez y J.L. Gonzalez; Nitrogen mineralization in soil with conventional and organomineral fertilization practices. Communications in Soil Science and Plant Analysis 33(19-20), 3679-3702 (2002).

Xiaoli C. y otros cuatro autores; Characteristics and mobility of heavy metals in an MSW landfill: Implications in risk assessment and reclamation. Journal of Hazardous Materials 144 (1-2), 485-491 (2007). 
